\title{
«Nur gemeinsam sind wir stark»
}

Interview: Felicitas Witte

Dr. med., Medizinjournalistin

Wenn die Ärzteschaft mit einer Stimme spricht, kann sie etwas bewegen. So lautet die Devise von Jacques de Haller, seit Anfang 2016 Präsident des Comité permanent des Médecins Européens (CPME). Gefordert wird eine effiziente interprofessionelle Zusammenarbeit und das Bewusstsein, wie bedeutend Ausbildung und ethisches ärztliches Handeln für eine optimale, europaweite Gesundheitsversorgung sind.

\section{SÄZ: Herr de Haller, warum sind Sie Präsident des permanenten Komitees Europäischer Ärzte CPME geworden?}

Das CPME ist die Dachorganisation der nationalen medizinischen Organisationen wie der FMH, der Bundesärztekammer in Deutschland oder des Ordre National der französischen Ärzte. Ich bin überzeugt, dass die Medizin und gut funktionierende Gesundheitssysteme einen wesentlichen Beitrag für eine gute Lebensqualität der Menschen leisten. Alles, was wir für eine gute Medizin machen können, sollten wir unterstützen - und natürlich auch auf europäischer Ebene.

\section{Was wollen Sie bei CPME erreichen?}

Ich möchte, dass Politiker uns Ärzte in Europa hören, damit sie mehr im Sinne unserer Weltanschauung handeln: eine humanistische und solidarische Vision der Welt, die auf unserer Erfahrung mit den Patienten basiert. Übrigens habe ich und meine Kollegen vom CPME-Vorstand kürzlich Stellungnahmen zu unseren Missionen und Visionen verfasst, die man online nachlesen kann ${ }^{1}$.

Auf der CPME-Homepage steht, beste Gesundheit und der Zugang zu einer Gesundheitsversorgung sollte für jeden Menschen Realität sein. Ist das nicht unrealistisch bei der derzeitigen finanziellen Situation vieler europäischer Länder?

Sicherlich stehen die Gesundheitssysteme der meisten europäischen Länder, vor allem der südeuropäischen, als Folge der Wirtschaftskrise unter einem enormen wirtschaftlichen Druck. Wir wollen uns dafür einsetzen, dass dieser Druck sich nicht auf die Patienten überträgt und dass jeder Mensch in Europa weiterhin jederzeit auf die nötige medizinische Hilfe zugreifen kann.

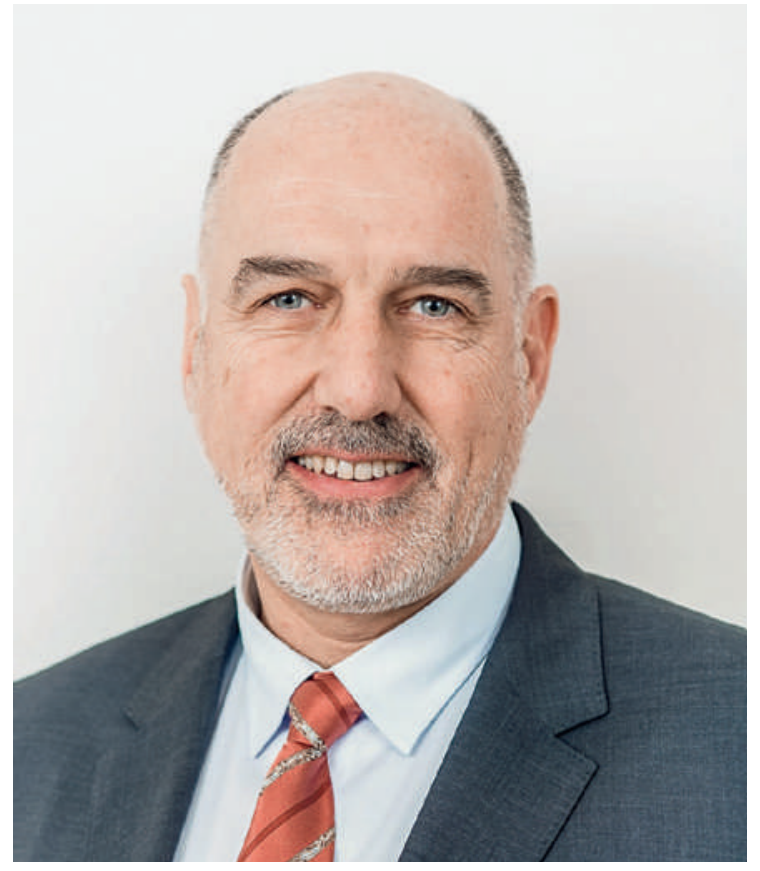

Jacques de Haller, Präsident des Comité permanent des Médecins Européens (CPME).

Das sind gute Vorsätze. Aber wie will CPME das erreichen?

Wir vertreten mehrere Millionen Ärzte und Ärztinnen. Das gibt uns in unseren Stellungnahmen, Diskussionen oder Briefen an Staatspräsidenten eine grosse Glaubwürdigkeit. Wie können die Welt natürlich total nicht ändern, haben aber sicher einen Einfluss.

\section{Welche Rolle spielt dabei der einzelne Arzt?} Was kann er tun?

Wir spielen eine unersetzliche Rolle für die Patienten, die auf unserer Einfühlsamkeit basiert, unserem Wissen und unserem ethischen Verständnis. Ethisch zu 
handeln bedeutet nicht nur, dass wir in "guten Zeiten» danach handeln. Den ethischen Gedanken müssen wir auch in "schlechten Zeiten" leben, jederzeit, selbst wenn wir unter politischem Druck stehen. Zwei CPMEMitglieder möchte ich hier erwähnen: In der Türkei sind Ärzte zurzeit mit der Gewalt des Staates konfrontiert, und in Israel stehen sie regelmässig unter dem Druck der Regierung. In der Türkei wurden Ärzte zum Beispiel erschossen, während sie Patienten auf der Strasse behandelten, die im Rahmen von Demonstrationen verletzt wurden. Den Ärzten wurde verboten, Demonstranten zu behandeln, und sie sollten jeden Tag der Polizei die Namen der Patienten nennen, die sie behandelt hatten. In Israel haben sich Ärzte geweigert, an Folter teilzunehmen, was ja auch selbstverständlich ist. Und sie haben sich geweigert, Gefangene zwangsweise zu ernähren. Das ist ebenfalls selbstverständlich und ethisch unabdingbar, aber für die Behörden und die Medien gar nicht so selbstverständlich. All das haben die Kollegen gemacht, obwohl sie unter massivem politischem Druck stehen. In beiden Ländern gelingt es den Ärzten also, ihre festen ethischen Standpunkte $\mathrm{zu}$ behalten und mit einer würdigen Haltung zu arbeiten.

\section{Welche Rolle spielt der Arzt-Patienten-Kontakt?}

Die Beziehung zwischen Arzt und Patient steht im Zentrum, ist der Kern der Medizin. Im Rahmen dieser Beziehung übertragen wir unsere Therapie. Es geht hier um einen Beziehungsraum, der unbedingt vor unrechtmässigen Einflüssen geschützt bleiben muss, etwa durch den Staat oder durch die Versicherer.

\section{Inwiefern kooperiert das CPME mit der FMH? Was ist Ihnen wichtig dabei?}

Die Schweiz ist Mitglied des Europäischen Freihandelsabkommens und an die Europäische Union durch zahlreiche bilaterale Vereinbarungen gebunden. So ist die FMH ein Vollmitglied des CPME. Wenn es um das Recht auf Personen-Freizügigkeit geht - direkt oder indirekt - wird in der Schweiz automatisch das europäische Recht angenommen. Die Themen, die wir im CPME besprechen, betreffen also oft direkt die Schweiz. Für jedes Thema kontaktiere ich das zuständige Mitglied im Zentralvorstand der FMH. Abgesehen davon werde ich sicherlich ein oder zweimal pro Jahr vom Zentralvorstand eingeladen, um die aktuellsten Themen zu diskutieren. Die Kollaboration mit der FMH ist sehr konstruktiv, ich freue mich darauf.

\section{Was haben die Schweizer Ärzte davon?}

Es ist natürlich für die Ärzte in der Schweiz von wesentlichem Vorteil, wenn ihre Organisationen sich früh genug in Diskussionen einschalten, die ihre Zukunft betreffen, zum Beispiel eHealth und Patientendossier, Diplomanerkennung oder Freizügigkeit. Oder die beruflichen Kompetenzen von Ärzten und Apothekern, also ob Apotheker zum Beispiel Medikamente ohne Rezept verkaufen und ob sie impfen dürfen. Unsere Stimme muss unbedingt gehört werden. Davon profitieren Kollegen in der Schweiz wie überall in Europa Ich betone nochmals, dass es nicht nur um Schweizer Ärzte geht, sondern um Ärzte überall in Europa, denn wir sind oft von den gleichen Problemen betroffen.

\section{Welche Themen haben Sie sich für Ihre Amtszeit vorgenommen und warum?}

Das sind im Grunde genommen die gleichen Themen, um die es in der Schweiz geht. Von den derzeitigen aktuellen gesundheitspolitischen Themen gibt es auf europäischer Ebene noch mehr als auf Schweizer Ebene. Die wichtigsten, um die ich mich in den folgenden Jahren kümmern möchte, betreffen die interprofessionelle Zusammenarbeit, die Selbst-Fürsorge der Patienten, also ihre Autonomie, die Autonomie der Ärzte, die Qualitätsgarantie und eHealth. All diese Themen haben mehr oder weniger mit der therapeutischen Freiheit der Ärzte zu tun. Diese Freiheit ist essentiell, um eine wirklich gute Medizin zu machen.

Warum ist eine bessere Kooperation zwischen Ärzten und nichtmedizinischen Berufen wichtig und wie wollen Sie das erreichen?

Eine optimale Zusammenarbeit zwischen den Berufsgruppen ist zunächst einmal wichtig, weil es zu einer besseren Qualität der Versorgung beiträgt und zum Wohlbefinden der Patienten. Wenn wir besser kooperieren, können wir zudem dem Mangel an Gesundheitsfachkräften besser begegnen. Schliesslich erlaubt es politisch eine stärkere Stimme, wenn wir gemeinsame Aktionen durchführen können.

Für so eine Kollaboration braucht es Einsatz, man muss sich mitunter über seine Gewohnheiten und Empfindlichkeiten hinwegsetzen. Man muss sich auch klar werden, was jeder Beruf für eine Identität und für Aufgaben hat und welche Rolle der Arzt oder der nichtmedizinische Mitarbeiter spielt. Indem wir miteinander sprechen, wird uns aber auch bewusst, dass der Patient eine Referenzperson braucht, und diese Person sollte meiner Meinung nach der Arzt sein.

\section{Mitunter hört man Klagen, die Arbeitsbedingungen} der Ärzte seien so schlecht. Muss man die verbessern? Gute Arbeitsbedingungen sind essentiell, um qualitativ hochwertige Medizin zu leisten. Man arbeitet zum Beispiel nicht gut, wenn man unter Zeitdruck oder 
Leistungsdruck steht oder viel zu viel arbeiten muss. Die Arbeitsbedingungen für uns Ärzte lassen in Europa zu wünschen übrig, ich bin damit nicht zufrieden. Ich könnte Ihnen von vielen Studien zur psychischen Gesundheit von Medizinern berichten - das ist erschreckend, und das gilt auch für die Schweiz. Wir müssen uns daher intensiv einsetzen, dass die Arbeitsbedingungen verbessert werden, um eine hohe Qualität der medizinischen Versorgung zu gewährleisten, die Gesundheit unserer Kollegen sicherzustellen und zukünftige Generationen für den Arztberuf noch begeistern zu können.

\section{Was kritisiert das CPME an europäischen Gesund- heitspolitikern? Was müsste sich ändern?}

Das europäische politische System funktioniert ähnlich wie die Schweiz, mit einer Exekutive, einem Parlament, Ausschüssen, Kommissionen, Konsultationen und Arbeitsgruppen. Die aktuelle Kommission von Jean-Claude Juncker spricht aber mit den Partnern aus
Dr. med. Felicitas Witte

Seefeldstrasse 285

CH-8008 Zürich

felicitas.witte[at]icloud.com dem Gesundheitssystem viel weniger als die vorherige Kommission, das bedauern wir im CPME sehr. Es wird ein langer und wichtiger Kampf sein, dass wir ausreichend gehört werden.

\section{Denken Sie, durch einen europäischen Ansatz kann} sich mehr ändern, als wenn die FMH beziehungsweise die Schweiz alleine Massnahmen trifft?

Das hängt natürlich von den Themen ab, aber ich bin überzeugt, dass wir gemeinsam mehr schaffen. Ich bin mir dagegen nicht sicher, ob die Schweiz - sei es die Bevölkerung insgesamt oder die verschiedenen Berufsgruppen - wirklich erkennt, dass sich jetzt ziemlich viele Bereiche des Lebens auch ausserhalb unserer Grenzen abspielen. Ich persönlich finde das gut und bereichernd, aber abgesehen davon: Es ist eine Realität, und es bringt nichts, diese zu ignorieren oder zu leugnen. Es wäre eine grosse Gefahr, weil wir damit die Kontrolle über unsere eigene Zukunft verlieren.

\section{Auf der Homepage vom CPME steht «European doctors call on EU decision makers». Gilt das auch für Entscheidungsträger in der Schweiz?}

Ich habe in der Schweizer Gesundheitspolitik mehr als 15 Jahre lang mit Begeisterung gewirkt. Ich habe versucht, das zu tun, was mir richtig erschien, und ich habe mich damit sehr wohl gefühlt. Jetzt arbeite ich aber an anderer Stelle; die Schweizer Gesundheitspolitik liegt in der Verantwortung der FMH und ihrer derzeitigen Führung. Ich kümmere mich nun in erster Linie um die europäische Politik.

\section{Wie können europäische Ärzte gemeinsam stark sein?} Dadurch, dass wir unsere Forderungen mit einer Stimme und quasi "aus einem Munde» stellen. So wie die Schweizer Ärzte durch eine Reihe gemeinsamer Grundsätze verbunden sind, sind es auch die europäischen Mediziner: ethisch und solidarisch zu handeln, den Patient und seine Interessen im Mittelpunkt des ärztlichen Tuns zu sehen und qualitativ hochwertige Medizin zu leisten. Diese Grundsätze vereinen Ärzte aus einem Land sowie aus einem Kontinent.

Es ist aber auch normal, dass sie je nach Kultur von Land zu Land mitunter anders umgesetzt werden. Das hindert uns aber nicht, gemeinsam stark zu sein.

Vielen Dank für das Gespräch. 